\title{
Large-Timestep Mover for Particle Simulations of Arbitrarily Magnetized Species
}

\author{
R. H. Cohen ${ }^{\text {a }}$ A. Friedman ${ }^{\text {a }}$ D. P. Grote ${ }^{a}$ J.-L. Vay ${ }^{b}$ \\ ${ }^{a}$ Lawrence Livermore National Laboratory, P.O. Box 808, Livermore, CA 94551 \\ $U S A$ \\ ${ }^{\mathrm{b}}$ Lawrence Berkeley National Laboratory, Berkeley, CA 94720 USA
}

\begin{abstract}
For self-consistent ion-beam simulations including electron motion, it is desirable to be able to follow electron dynamics accurately without being constrained by the electron cyclotron timescale. To this end, we have developed a particle-advance that interpolates between full particle dynamics and drift motion. By making a proper choice of interpolation parameter, simulation particles experience physically correct parallel dynamics, drift motion, and gyroradius when the timestep is large compared to the cyclotron period, though the effective gyro frequency is artificially low; in the opposite timestep limit, the method approaches a conventional Boris particle push. By combining this scheme with a Poisson solver that includes an interpolated form of the polarization drift in the dielectric response, the movers utility can be extended to higher-density problems where the plasma frequency of the species being advanced exceeds its cyclotron frequency. We describe a series of tests of the mover and its application to simulation of electron clouds in heavy-ion accelerators.
\end{abstract}

Key words: particle simulation, ion beams, accelerators, plasmas PACS: 29.27.-a, 07.05.Tp, 52.65.-y

\section{Introduction}

For simulations of ion beams in magnetically focussed accelerators which selfconsistently include the effects of electron clouds, it is necessary to follow electrons through regions in which they are strongly magnetized (cyclotron frequency $\omega_{c e}$ large compared to other inverse timescales of interest) as well

Email address: rcohen@llnl.gov (R. H. Cohen). 
as regions where they are unmagnetized. It is desirable to have a simulation approach that is not constrained by the electron cyclotron timescale.

There are numerous other examples of systems in which particles transit regions of strong and weak magnetic field, yet the cyclotron motion itself is not of particular interest. These include field-reversed configurations for magnetic fusion, reconnecting plasmas with field nulls, and systems with self-generated strong localized magnetic fields.

To address this issue, we have developed a "blended particle mover" that interpolates between full particle dynamics and drift-kinetic dynamics. This mover was described briefly, and some tests presented, in a previous publication [1]. In this paper we describe the mover in more detail, present additional test results, and also describe an extension to the original prescription that allows extension to some higher-density systems with plasma frequency $\omega_{p}$ exceeding the gyrofrequency (along with a test of the new capability).

We note that, in addition to the original motivating applications, which can be generically described as problems with strongly varying magnetization, the blended mover concept may provide an attractive alternative to gyrokinetics for simulating magnetized plasmas.

\section{Blended mover}

The blended mover concept [1] builds upon the observation by Parker and Birdsall[2] that the conventional Boris mover for particles in a magnetic field, when directly applied with timesteps $\Delta t$ large compared to the inverse cyclotron frequency $\omega_{c}^{-1}$, recovers physically correct electric and magnetic drifts, at least for test problems where these drifts are uniform, but with two drawbacks: (1) the particles oscillate about their gyrocenter with an effective "gyroradius" that is larger than the physical gyroradius by a factor of $\left[1+\left(\omega_{c} \Delta t / 2\right)^{2}\right]^{1 / 2}$, and (2) the particles oscillate by nearly $\pi$ in gyrophase each timestep, with a slow procession period that is $\mathcal{O}\left(\omega_{c} \Delta t^{2}\right)$. For simulation of phenomena with frequencies well below the cyclotron frequency, the first difficulty can be overcome by using an interpolated velocity to advance particle positions. The instantaneous velocity $v_{L}$ is updated according to

$$
\mathbf{v}_{L, \text { new }}=\mathbf{v}_{L, \text { old }}+\left[(\Delta \mathbf{v})_{\text {Lorentz }}+(1-\alpha)(\Delta \mathbf{v})_{\mu \nabla B}\right]
$$

where the first term on the right hand side is the standard Boris update for the full velocity, and the second term is a mirror-force correction term which is discussed and defined below. The particle position is updated using an effective 
velocity which is an interpolation of this updated instantaneous velocity and the drift velocity $\mathbf{v}_{d}$; specifically, $\mathbf{x}^{n+1}=\mathbf{x}^{n}+\mathbf{v}_{\text {eff }}^{n+1 / 2} \Delta t$, with:

$$
\mathbf{v}_{e f f}=\mathbf{b}\left(\mathbf{b} \cdot \mathbf{v}_{L}\right)+\alpha \mathbf{v}_{L, \perp}+(1-\alpha) \mathbf{v}_{d}
$$

and $\mathbf{v}_{d}$ is the sum of the particle $\mathbf{E} \times \mathbf{B}, \nabla B$, and curvature drifts. The logic behind the blended scheme is that adding a fraction $\alpha$ of the oscillating perpendicular (to $\mathbf{B}$ ) velocity to the position advance will result in an oscillation about the field line that is reduced by a factor $\alpha$. From the expression above for the effective gyroradius of the Boris mover, it follows that the choice $\alpha=1 /\left[1+\left(\omega_{c} \Delta t / 2\right)^{2}\right]^{1 / 2}$ produces the physically correct gyroradius. Furthermore, for any choice of $\alpha$, since the Boris mover reproduces correct parallel dynamics and particle drifts, at least for the simple cases considered by Parker and Birdsall,and, by construction, so does drift kinetics, the interpolation given in Eq. (2) will as well. In fact it can be expected to do better than either pure Boris or drift kinetics for cases where there is significant nonlinear variation of the local particle drift on the scale of the gyroradius, as the blended scheme will average the drifts over the proper gyroradius.

The magnetic-mirror force $\mu \nabla B$ arises from the local convergence or divergence of field lines. It is properly calculated in a full orbit dynamics calculation, but needs to be explicitly added to the parallel dynamics in the drift limit. Hence it appears multiplied by the complement of the interpolation function in Eq. (1). Since it is a magnetic force it conserves energy, and so can be represented as a rotation of the velocity $\mathbf{v}_{L}$ in the plane defined by $\mathbf{v}_{L}$ and the magnetic field, by an (assumed small) amount $\Delta \theta=\left(m \mu / 2 B^{3}\right)^{1 / 2} \Delta t \mathbf{B} \cdot \nabla B$ where $\mu \equiv m v_{\perp}^{2} / 2 B$.

This algorithm was implemented into the explicit electrostatic code WARP[3], utilizing a simple predictor-corrector scheme for particle advance only: to advance from time level $n$, Eq. (1) is advanced from level $n-1 / 2$ to $n+1 / 2$ using fields at time level $n$, and, in the evaluation of $(\Delta \mathbf{v})_{\mu \nabla B}$, the old particle velocities. The position is advanced uncentered from time level $n$ to $n+1$ and the average of this predicted position and the position at level $n$ is used to construct effective drift velocities at level $n+1 / 2$.

\section{Test problems}

The mover described above has been subjected to a number of tests of increasing complexity. First, single-particle electron orbits were calculated in a quadrupole magnetic field, with a superimposed stationary positive charge

distribution (mocking up the effect of an ion beam). Orbits that pass too close 

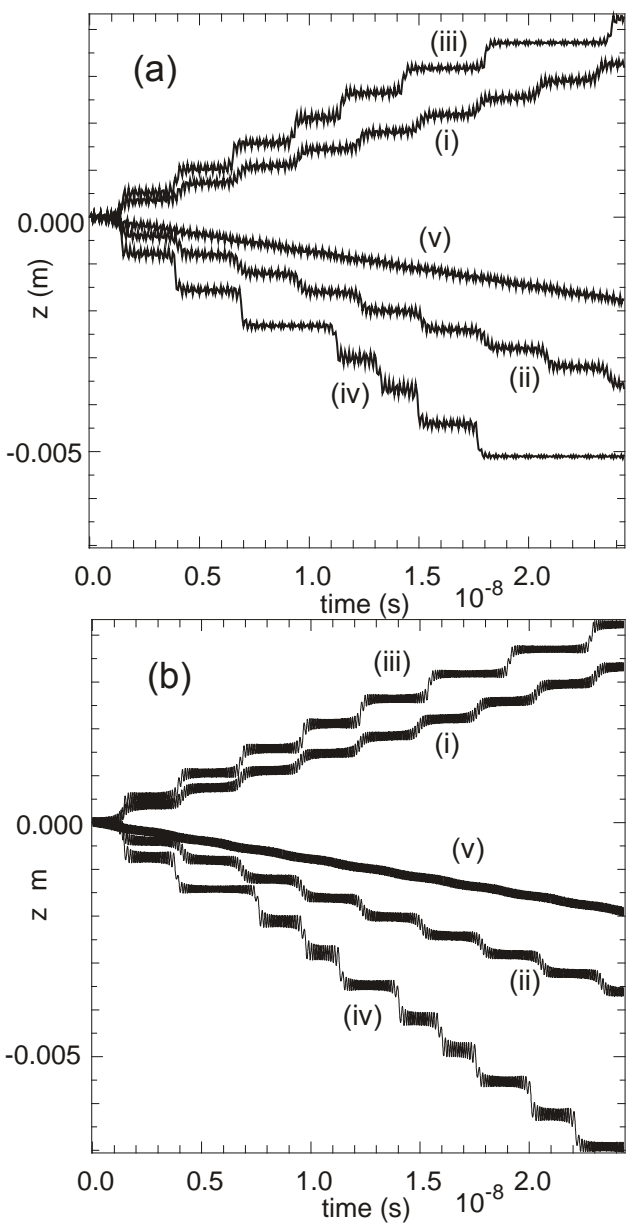

Fig. 1. Single-particle orbits in quadrupole field with (a) $\omega_{c, \max } \Delta t=5 \pi$ and (b) $\omega_{c, \max } \Delta t=0.2 \pi$. Particles were initialized at azimuthal angles $\theta=$ (i) $40^{\circ}$, (ii) $50^{\circ}$, (iii) $43^{\circ}$, (iv) $47^{\circ}$, and (v) $70^{\circ}$. The quadrupole is oriented so that the principal axes are at $\theta=45^{\circ}$ and $135^{\circ}$.

to the field null in such a system experience chaotic, nonadiabatic changes in magnetic moment. A range of initial conditions were chosen to obtain orbits that were adiabatic, marginally nonadiabatic, and strongly nonadiabatic, and the axial positions versus time plotted, as shown in Fig. 1. This was done for a large-timestep simulation $\left(\omega_{c, \max } \Delta t=5 \pi\right)$ and a small timestep simulation which resolves the maximum cyclotron frequency $\left(\omega_{c, \text { max }} \Delta t=0.2 \pi\right)$. The steps in orbits (i), (ii), (iii), (iv) occur as electrons pass close to the field null and experience a large magnetic curvature drift; their spacing is an indication of the bounce time. Adiabatic orbit (v), and marginally nonadiabatic orbits (i) and (ii), agree very well; orbits (iii) and (iv) are chaotic, as evidenced by the variability in bounce time, and hence agreement is not expected (except that both small and large timestep simulations exhibit the chaotic behavior, and a very small change in the initial conditions produces a significantly different result). 


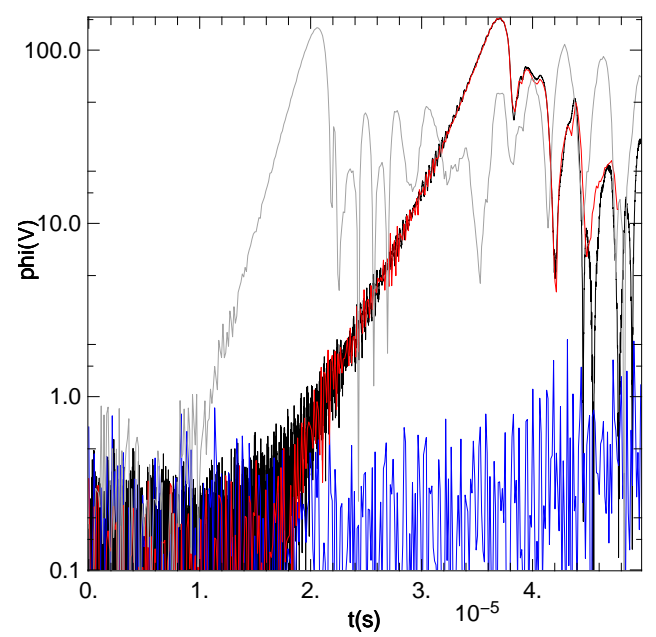

Fig. 2. Growth rate for two stream instability of finite-size beams. Black curve is 10-gyroadius beam using small timesteps; red is same beam using large timesteps and blended mover; blue is same beam using large timesteps and pure Boris mover; gray curve is 20-gyroradius beam using large timesteps and blended mover.

A second test is the calculation of a two-stream instability for two colliding finite-size (10 gyroradius) anisotropic $\left(T_{\perp} \gg T_{\|}\right)$proton beams. We compare results from simulations at small timestep $\left(\omega_{c} \Delta t=0.6\right)$, large timestep $\left(\omega_{c} \Delta t=12\right)$ using the blended mover, and large timestep using the pure Boris mover. This test was reported in Ref. [1], though we have since discovered that the code at the time had an inconsistent boundary condition. Correcting that produces quantitative but not qualitative changes. In Fig. 2 we show the time evolution for the corrected simulation, with the results for the blended mover slightly shifted horizontally to subtract out the difference in the effective noise seed in the large-timestep. The results clearly illustrate that the blended mover recovers the correct linear growth and even closely tracks details of the early evolution of the saturated state. To illustrate the importance of the finite beam radius, a comparison case with a double-radius beam is also shown (gray curve).

A third test, also discussed in Ref. [1] is the calculation of electron cloud dynamics for a self-consistent simulation which includes both an ion beam and electrons. This is a simulation of an experiment done in the High Current Experiment (HCX) facility[4], in which an ion beam which has passed through is series of quadrupole magnets and a magnetic-field-free end region is allowed to impact an end plate which emits a copious supply of electrons. Shown in Fig. 3, taken from Ref. [1], is a plot of the electron distribution in the final quadrupole magnet and field-free region for the blended mover at large timestep and a comparison run with small timesteps. The blended mover does a good job of reproducing the electron distribution calculated with small timesteps, including capturing the spatial oscillations which appear. These oscillations propagate upstream (relative to the ion beam propagation) with the 

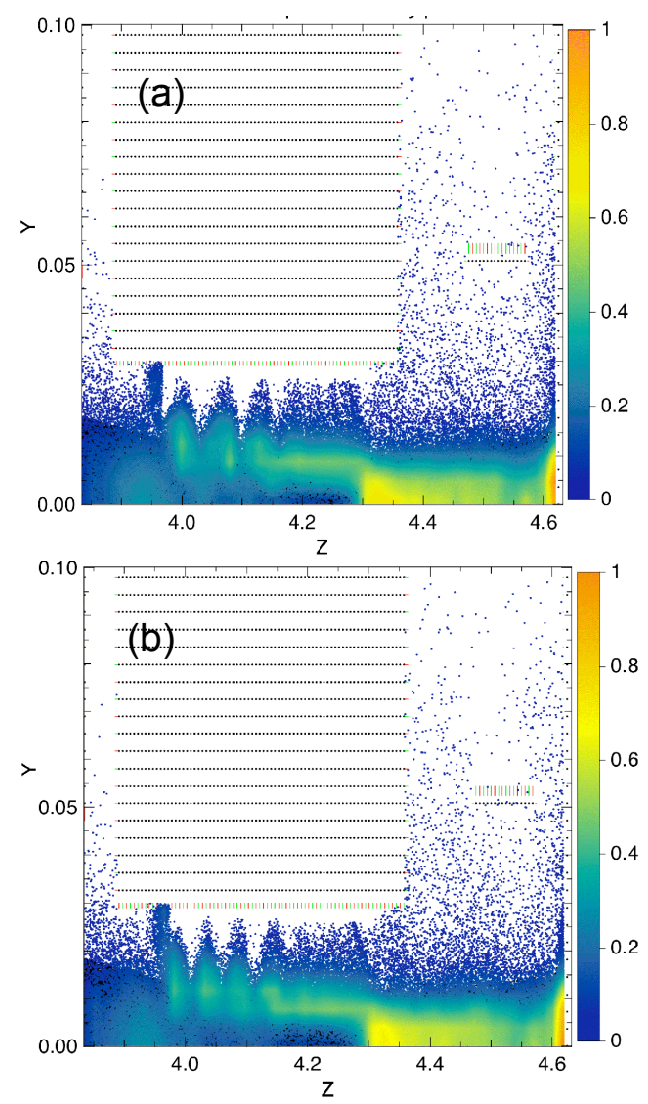

Fig. 3. Electrons in self-consistent electron-ion simulation of 4th magnet of HCX, using (a) the blended mover and large timesteps, $\omega_{c} \Delta t \approx 2 \pi$, and (b) the Boris mover with small timesteps, $\omega_{c} \Delta t \approx 2 \pi / 10$

drifting majority electron population, and hence would appear as temporal oscillations on a stationary probe. Not shown in Fig. 3 is a simulation done with the pure Boris mover and the large timestep, which is qualitatively different, in particular missing most of the oscillation.

A fourth test, to be described in detail elsewhere[5], entails the calculation of the spatial distribution of positrons in a Penning-Malmberg trap with an added mirror coil, using the blended mover. The results agree well with predictions of an analytic model.

Validation tests of the simulation capability, including the blended mover, has been done by comparing experimental signals from the HCX experiment with synthetic diagnostics in the WARP code. In particular the electron current collected on a biased electrode upstream of the last quadrupole magnet has been measured and also simulated; the results are shown in Fig. 4. It is seen that the simulation reproduces the magnitude and characteristic frequency range of the experiment. 


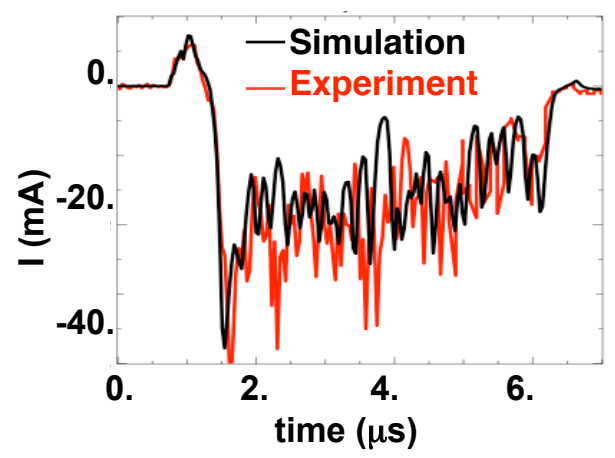

Fig. 4. Comparsion of collected current on electrode in HCX experiment and signal synthesized from WARP simulation.

\section{High-density extension}

The blended mover implementation described above is limited to problems where the species to which it is applied is low density, as characterized by the plasma frequency being less than the cyclotron frequency. The mover is of benefit only if it allows timesteps larger than the cyclotron period, but the existing explicit implementation limits the timestep to be at most of order of the inverse of the plasma frequency $\omega_{p}$.

We have taken two steps to remove these restrictions: (1) incorporation of the polarization drift as an additional term in the Poisson equation; this term becomes an order unity correction when $\omega_{p} \sim \omega_{c}$; and (2) implementation of a centered predictor-corrector scheme that includes a field solve at the end of the predictor step. With these changes we can address high-density problems where geometrical constraints preclude formation of a simple one-dimensional (along B) plasma wave. The inclusion of polarization in the Poisson equation, coupled with the modified predictor-corrector scheme, introduces a degree of implicitness sufficient to allow solution of such problems.

The generalized Poisson equation follows from a determination of the charge density perturbation produced by the polarization drift[6], and can be written in the form

$$
\nabla^{2} \phi+\sum_{s} \nabla_{\perp}\left(\omega_{p s}^{2} / \omega_{c s}^{2}\right) \nabla_{\perp} \phi=4 \pi e\left(n_{i}-n_{e}\right)
$$

where $\nabla_{\perp}=\nabla-\mathbf{b b} \cdot \nabla$ is the gradient perpendicular to the magnetic field and $n_{e}$ and $n_{i}$ are the electron and ion densities calculated without inclusion of the polarization drift, and where the sum $s$ runs over species. The second term on the left is the polarization response. Since the polarization drift is 
intrinsically included in the direct Boris particle advance and so in the density calculated from the direct particle advance, for the blended mover the above equation must be corrected. The density on the right-hand side of a species pushed with the blended mover will contain a fraction $\alpha$ of the polarization density, corresponding to the fraction of the Boris move used in the particle advance for species $s$. Hence the polarization response for species $s$ should be multiplied by $\left(1-\alpha_{s}\right)$. Hence the appropriate form of the Poisson equation becomes:

$$
\nabla^{2} \phi+\sum_{s}\left(1-\alpha_{s}\right) \nabla_{\perp}\left(\omega_{p s}^{2} / \omega_{c s}^{2}\right) \nabla_{\perp} \phi=4 \pi e\left(n_{e}-n_{i}\right)
$$

and the sum runs only over species for which the blended mover is used.

The other change is the implementation of a centered predictor-corrector scheme. To advance from time level $n$ to $n+1$, we advance Eq. (1) from $n-1 / 2$ to $n+1 / 2)$ as in Sec. 2, and then advance particle positions from time level $n-1$ to $n+1$ using the effective velocity from Eq. (2), with field quantities evaluated at time level $n$, and instantaneous velocities $v_{L}$ averaged over levels $n-1 / 2$ and $n+1 / 2$. A field solve is then performed for particles at the predicted positions. A corrector step is then taken from step $n$ to $n+1$, using $v_{L}^{n+1 / 2}$ and the average of the drift velocities at level $n$ and those calculated from the predicted particle locations and fields. The corrector step can be repeated multiple times if needed.

As a test problem we consider a simple plasma instability, the slab ion temperature gradient instability in two dimensions [7]. This instability occurs in a plasma with a uniform magnetic field provided that $\eta_{i}$, the logarithmic temperature gradient divided by the logarithmic density gradient, exceeds a critical value. This instability was calculated using gyrokinetics by Lee and Tang[7]. Lee and Tang developed a multiscale approach[8] to including the temperature and density gradient drive terms that allowed them to perform simulations with a uniform plasma in a doubly periodic box; specifically they add to the particle drifts a fake compressible term

$$
\Delta \mathbf{v}=\left(\kappa_{n}-\left(3 / 2-v^{2} / v_{t h}^{2}\right) \kappa_{T}\right) \mathbf{e}_{x} \times \mathbf{B} / B
$$

and where $\kappa_{n}=-(1 / n) d n / d x$ and $\kappa_{T}=-(1 / T) d T / d x$ to the velocities used to advance the particles. We apply the same approach here, adding it to $v_{\text {eff }}$. We test here application of our mover to ion dynamics, with the electrons treated by an adiabatic approximation, $n_{e}=\hat{n} \exp (e \phi / T)$, with $\hat{n}$ a constant on a field line, which we choose to be $n_{0} /\langle\exp (e \phi / T)\rangle$ where $n_{0}$ is the initial (constant) density and \langle\rangle denotes a field-line average. This is a nonlinear extension of a linear model commonly used for gyrokinetic simulation, which preserves the number of electrons on a field line. 

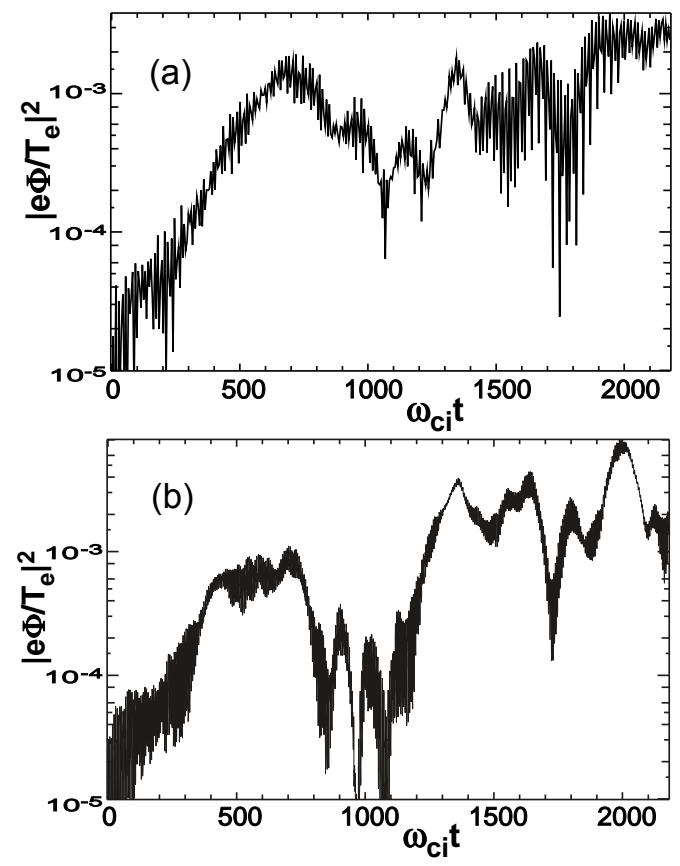

Fig. 5. Growth of the potential for the slab ion-temperature gradient mode, for the (1,1) Fourier component, obtained with adiabatic electrons and using (a) the blended mover, and (b) the standard Boris mover with a smaller timestep $\left(\omega_{c i} \Delta t=0.25\right)$

We have run the specific problem described in Ref. [7]: a two-dimensional slab $x, z$, with the magnetic field in the $y, z$ plane and $B_{z} / B_{y}=0.01$, with cell size $=$ gyroradius, $\kappa_{n}=0.05$ or $0, \kappa_{T i}=0.2, \omega_{p i} / \omega_{c i}=\left(m_{i} / m_{e}\right)^{1 / 2}$. We run with the blended mover with $\omega_{c i} \Delta t=5.43$. Two iterations of the corrector are required for numerical stability [as determined by running a problem with the drive term (5) turned off]. To reduce noise we follow the common practice of smoothing the charge density (with a 1-2-1 filter) prior to solving Poisson's equation. The resulting potential solution is Fourier-analyzed in the two spatial dimensions. The time history of the resultant dominant $n_{x}=n_{z}=1$ mode is plotted in Fig. 5 for $\kappa_{n}=0$ The plots show linear growth followed by saturation at levels comparable to those shown in Ref. [7] and those found with other gyrokinetic codes [9]. The oscillations in the saturated phase are comparable in their period to those observed by Lee and Tang. We infer a linear growth rate from fitting this data (and dividing by two to get the growth rate for $\phi$ itself, of approximately $0.004 \omega_{c i}$, which is about $30 \%$ more than the simulation result obtained by Lee and Tang, but still less than the fluid-based analytic result which Lee and Tang use as a basis for comparison. We have also analyzed the $n_{x}=1, n_{z}=-1$ mode; it has a similar growth rate and saturation level. We have also run the model with other choices for $\hat{n}$, namely $\hat{n}=n_{0}$ and $\hat{n}=\left\langle n_{i}\right\rangle /\langle\exp (e \phi / T)\rangle$, and the results are similar.

In the test problems reported in Sec. 3, blended-mover results were compared 
with those using the Boris mover at both small and large timesteps. We show the comparison with a small-timestep run $\left(\omega_{c i} \Delta t=0.25\right)$ in Fig $5 \mathrm{~b}$; the result is sufficiently noisier than the blended mover that it is impossible to obtain a clean estimate of the linear growth rate, but it and the saturation level can be seen to be roughly comparable to that for the large-timestep blended mover. The Boris mover run at the large timestep described above is unstable (large potentials grow even in the absence of gradients).

\section{Discussion}

The blended mover described here allows simulation of particles in strongly inhomogeneous magnetic fields without being constrained by the maximum cyclotron frequency. it is quite successful in reproducing results from simulations that resolve the cyclotron motion, but with a much larger timestep. It reproduces single-particle drifts and parallel dynamics quite well, even to the extent of correctly distinguishing chaotic and non-chaotic orbits near the null of a quadrupole field (reproducing the trajectory for non-chaotic orbits). It allows us to recover results for an electron collective oscillation that reproduce results from both short-timescale simulation and from experimental data. This mover is now in routine use in the WARP particle code.

The modified implementation of the mover discussed in Sec. 4 was introduced to allow application to higher-density problems where $\omega_{p}>\omega_{c}$. Our tests indicate that it in fact does that. Success depended on inclusion of the polarization term in the Poisson equation, which operates only on variations perpendicular to the magnetic field. Removal of this restriction requires a fully implicit implementation, which we plan to do. Particularly with these high-density extensions, the mover is a potential alternative to gyrokinetics for simulating kinetic phenomena in strong magnetic fields while retaining finite gyroradius effects.

\section{Acknowledgments}

We thank Drs. B. I. Cohen and A. M. Dimits for valuable discussions. This work was performed under the auspices of the U.S. Department of Energy by University of California Lawrence Livermore National Laboratory under

contract W7405-ENG-48 and by University of California Lawrence Berkeley National Laboratory under contract DE-AC03-76F00098. 


\section{References}

[1] R. H. Cohen, A. Friedman, M. Kireeff Covo, S. M. Lund, A. W. Molvik, F. M. Bieniosek, P. A. Seidl, J.-L. Vay, P. Stoltz, and S. Veitzer, Phys. Plasmas 12, 056708 (2005).

[2] S. E. Parker and C. K. Birdsall,J. Comp. Phys 97, 91 (1991)

[3] D. P. Grote, A. Friedman, I. Haber, Fus. Eng. \& Des., 32-33, 193 (1996) and http://hif.lbl.gov/theory/WARP_summary.html

[4] P. A. Seidl, D. Baca, F. M. Bieniosek, C. M. Celata, A. Faltens, L. R. Prost, G. Sabbi, W. L. Waldron, R. Cohen, A. Friedman, S. M. Lund, A. W. Molvik, Proceedings 2003 Particle Accelerator Conference, paper ROAC001 (IEEE, Piscataway, NJ, 2003);

http://accelconf.web.cern.ch/AccelConf/p03/PAPERS/ROAC001.pdf

[5] K. Gomberoff, J. Fajans, J. Wurtele, A. Friedman, D. P. Grote, R. H. Cohen and J.-L. Vay, "Simulation studies of non-neutral plasma equilibria in an electrostatic trap with a magnetic mirror", submitted to Phys Plasmas (2006).

[6] See, e.g., C. L. Longmire, Elementary Plasma Physics [Interscience, New York, 1963], p. 31.

[7] W. W. Lee and W. M. Tang, Phys. Fluids 31, 612 (1986).

[8] W.W. Lee, J. Comput. Phys. 72, 243 (1987).

[9] B. I. Cohen, private communication (2006). 